\title{
A I IST OF THE MOSSES, LIVERWORTS AND LICHENS OF ILLINOIS.
}

\author{
By JOHN WOLF and ELIHU HALL.
}

The specimens upon which the following list is based were nearly all collected by the writers; those from Menard county by Mr. Hall, those from Fulton county by Mr. Wolf. Those from Southern Illinois were mostly obtained by Mr. Wolf, as botanical collector of a party from the State Laboratory which visited the counties of Union, Johnson and Jackson, during July and August, 1877.

We are under great obligation to Prof. Leo Lesquereux, Thomas P. James, Esq., Coe F. Austin, Esq., and Henry Willey, Esq., for the determination of species, and for many other favors.

\section{IMUSCI.}

Archidium, Brid.

A. ohioense, Schimp.,

Menard.

Ephemerum, Hampe.

E. crassinervium, Schwægr.,

Fulton, Menard.

Acaulon, Mull.

A. triquetrum, Spruce.,

A. muticum, Schreb.,

A schimperianum, Sull.,

A. wolfii, James,

Menard.

Fulton.

Menard.

Fulton.

Phascum, $L$.

P. euspidatum, Schreb.,

Fulton, Menard.

Pleudidium, Brid.

P. alternifolium, Brid.,

Fulton, Menard. 
Astomum, Ilampe.

A. crispum, Hedw.,

A. sullivanti, Sclimp.,

A. nitidulum Schimp.,

Fulton.

Menard.

Fulton, Meuard.

Bruchia, Schwegr.

B. flexuosa, Schwirgr,

B. beyrichiana, Humpe.,

Fulton.

IIl.

Weisia, $/ e d w$.

W. viridula, Brid.,

W. mucronata, Br. \& Schf.,

Fulton, Menard. Fulton.

Campylopus, Brill.

C. leanus, Sulliv.,

Fulton, Meuard.

Trematodon, Rich.

T. longicollis, Rich.,

Union, Johnson.

Dicranum, Hedu.

D. varium, Hedw.,

D. rufescens, Turner,

D. heteromallum, Hedw.,

D. flagellare, Heduc,

D. scoparium, $L$.,

D. palustre, Brid.,

D. undulatum, Turner,

D. spurium, Hedu., var, condensatum,

Fulton, Menard. $66 \quad 66$

$66 \quad 66$

Fulton, Menard, Jolinson. Fulton, Menard.

Ill.

Menard.

Menard.

Ceratodon, Brid.

C. purpureus, Brid.,

Fulton, Menard, Lee'

Leucobryum, Hiempe.

L. glaucum, Hampe,

L. minus, Hampe,

Fulton, Menard. Johuson.

Fissidens, Hedu.

F. obtusifolius, Wils.,

F. miuutulus, Sullic.,

F. bryoides, IIcdu.,

F. subbasilaris, Hcdw.,

F. taxifolius, Hertw.,

F. adiantoides, Hed $u$.,

Fulton. Fulton, Menard. Fulton, Menard, McHenry. Fulton, Ilenard.

66 6

Fulton, Kane, Menard.

Cononitrium, Montagne.

C. julianum, Wont.,

C. ballianum, Sulliv. \& Lesqx.,

Fulton, Menard. Menard. 
Trichostomum, $B r . \& S c h$.

T. tortile, Schrad.,

T. pallidum, Hedw.,

T. tophaceum, Brid.,

T. rigidulum, Smith,

Fulton, Menard.

Fulton, Menard, Union, Johnson.

III.

Ill.

Barbula, Hedw.

B. unguiculata, Hedw.,

Menard.

B. caespitosa, Srhwogr,

Fulton, Menard, S. Ill.

Menard.

B. papillosa, Wils., 66

B. rigida, Schultis,

Fulton.

B. fallax, Hedu.,

B. vinealis, Brid.,

Menard.

B. subulata, Brid.,

Ill.

Desmatodon, Brid.

D. flavicans, Br.\& Schimp.

Peoria Co.

Didymodon, Br.\& Sch.

D. rubellus, $B r$. S Sch.,

Fulton.

PоттіA, Ehrh.

P. subsessilis, Br.\& Sch.,

Menard.

P. exigua, Aust.,

Tetraphis, Hedw.

T. pellucida, Hedw.,

Menard.

Encalypta, Schreler.

E. ciliata, Hedu.,

Ogle.

Drummondia, Hook.

D. clavellata, Hook,

Fulton, Menard.

Orthotrichum, Hedw.

O. strangulatum, Beauv.,

O. canadense, Br. \& Sch.,

O. crispulum, Hornsch,

Fulton, Menard.

Fulton.

Fulton, Menard.

Ptychomitrium, Br.\& Sch.

P. drummondi, Hook. \& Wils.,

S. III.

Grimmia, Ehrh.

G. apocarpum, $B r . \& S c h$,

G. confertum, Br. \& Sch.,

Menard.

G. ovata, $V . \& M$. 
Hedwiga, Ehrh.

H. ciliata, Ehrh.,

Tulton, Menard.

Atrichind, Beaut.

A. undulatum, Beauv.,

A. angustatum, Beauv.,

Fulton, Menard. Fulton, Menard, Johnson.

Pogonatum, Beaur.

P. brevicaule, Brid.,

Fulton, Menard.

Polytrichum, Brid.

P. commune, Linn.,

P. formosum, Hedu.,

P. juniperinum, Hedu.,

Fulton, Menard. Menard, Union. Fulton, Menard.

Timma, Iledu.

T. megapolitana, Hedw.,

Fulton, Menard.

Aulacominion, schwegr.

A.'heterostichum, Br. \& Sch.,

Fulton, Nenard.

Bryum, Bis \& Sch.

B. pyriforme, Hertu.,

B. nutans, Schreb.,

B. roseum, Schreb.,

B. albicans, Wahl,

B. argenteum, Limn.,

B. cernuum, Hedu.,

B. bimum, Schrel.,

B. intermedium, Brid.,

B. caespiticium, $L$.,

B. atropurureum, Web.\& Mohr.,

B. obconicum, Hsch.,

B. uliginosum, Br. \& Sch.,

Fulton, Menard.

Fulton, Kane, Menard. Fulton, Menard.

$\begin{array}{cc}66 & 66 \\ 66 & 66 \\ 66 & 66 \\ 66 & 66 \\ 66 & 66 \\ 66 & 66 \\ & \text { Menard. } \\ & \text { Fulton }\end{array}$

Mnium, $\mathrm{Br}$ \& Sch.

M. affirie, Blund,

M. punctatum, Hedu.,

M. serratum, Brid.,

N. stellare, Hedw.,

M. cuspidatum, Hedur.,

Fulton, Menard. Menard.

66

66

Fulton, Menard.

Bartrania, Hedu.

B. pouniformis, Hedu.,

B. fontana, Brid.,

B. marehica, Brid.,

B. radicalis, Beauv.,

Fulton, Menard.

Nenard.

Fulton.

Menard. 
Funaria, Schreb.

F. hygrometrica, Hedw.,

Fulton, Menard, N. III. Menard.

F. flavicans, Michx.,

F. microstoma, $B r . \&$ Sch.,

Discelium, Brid.

D. nudum, Brid.,

Fulton.

Physcomitrium, Brid.

P. pyriforme, Br.\& Sch.,

P. immersum, Sull.,

Fulton, Menard.

III.

Aphanorrhegma, Sulliv.

A. serratum, Sulliv.,

Fulton, Menard.

Fontinalis, Dill.

F. biformis, Sulliv.,

F. filiformis, $S . \& L$.,

F. dalecarlica, Bryol. Europ.,

Fulton.

Fulton, Mason.

Fulton.

Dichelyma, Myrin.

D. capillaceum, Bryol. Europ.,

Fulton.

LEUCODON, Schwægr.

L. julaceus, Sulliv.

Fulton, Menard, S. Ill.

Leptodon, Mohr.

L. trichomitrion, Web., var. immersum, $S . \& L$.,

L. ohioense, Sulliv.,

Menard, S. IIl. Pulaski.

Anomodon, Hook. \& Tayl.

A. obtusifolius, $B r . \&$ Sch.,

A. attenuatus, Hub.,

A. tristis, Cesati,

A. rostratus, Hedw.,

Fulton, Menard.

$66 \quad 66$

$66 \quad 66$

Fulton, Menard, S. Ill.

Leskea, Hedw. ; Bryol. Europ.

L. polycarpa, Hedw.,

L. obscura, Hedw.,

Fulton, Menard, S. Ill.

L. denticulata, Sulliv,

L. austini, Sulliv., Fulton.

III.

Fulton, Menard.

Clasmatodon, Hook. \& Wils.

C. parvulus, Hampe,

Fulton. 
Thelia, Sulliv.

T. hirtella, Heclw.,

T. asprella, Schimp.,

T. lescurii, Sulliv.,

$$
\begin{array}{r}
\text { Menard. } \\
\text { Fulton, Menard. } \\
\text { Fulton. }
\end{array}
$$

Fabronia, Racldi.

F. gymnostoma, Sullic. \& Lesqx.,

Fulton, Menard.

Anacamptodon, Bricl.

A. splachnoides, Brid.,

Fulton, Menard, S. Ill.

Pylaisaea, Bryol. Europ.

P. subdenticulata, W. P. Sch.,

P. intricata, Bryol. Europ.,

P. polyantha, Schreb.,

Fulton, Menard.

$66 \quad 66$

Fulton.

Homalothecium, Bryol. Europ.

H. subcapillatum, Bryol. Europ.,

Fulton, Menard.

Platygyrium, Bryol. Europ.

P. repens, Bryol. Europ.,

Fulton, Menard.

Cylindrothecium, Bryol. Europ.

C. cladorrhizans, Bryol. Europ.,

C. seduetrix, Bryol. Europ.,

C. compressum, Bryol. Europ.,

C. brevisetum, Bryol. Europ.,

Fulton, Menard.

$66 \quad 66$

$66 \quad 66$

Menard.

Cumacium, Web. \& Wohr.

C. americanum, Bricl.,

Fulton, Menard.

Hypnum, Dill.

H. delicatulum, Hedw.,

H. minutulum, Hedu.,

H. scitum, Beauv.,

H. gracile, Br. \& Sch.,

H. triquetrum, $L$.,

H. alleghaniense, C. Mull.,

H. hians, Hedx.,

H. novaeangliae, $S . \& L$.

II. sullivanti, Spruce,

H. diversifolium, Schimp,

H. strigosum, Hoffm.,

H. boseii, Schwacegr,

H. serrulatum, Hedwe,

H. deplanatum, W. P. Sch.,

Fulton, Menard.

$66 \quad 66$

$6 \quad$ i6

Fulton, Kane, Menard.

Fulton, Menard.

S. Ill.

Fulton, Menard.

Ill.

Fulton, Menard.

Menard.

Marion, Menard.

Menard.

Fulton, Menard.

66

66 
H. microcarpum, C. Mull.,

H. micans, Schwartz,

$$
\text { var. albulum, (C.M.) Aust., }
$$

H. cylindrocarpum, C. Mull.,

H. schreberi, Willd.,

H. aduncum, Hedw.,

H. crista-castrensis, $L$.,

II. imponens, Hedlw.,

H. curvifolium, Hediw.,

II. haldanianum, Grev.,

H. collinum, $B r$. \& Sch. $f$,

H. salebrosum, Hoff.,

H. laetum, Brid.,

H. acutum, Mitten,

H. acuminatum, Beauv.,

H. rivulare, Bryol. Europ.,

H. chrysophyllum, Brid.,

H. hispidulum, Brid.,

H. sommerfeldtii, Myrim,

H. dimorphum, Brid.,

H. adnatum, Hedw.,

H. serpens, Hedw.,

var. radicale, Brid.,

var. orthocladon, Beauv.,

H. riparium, $H_{e} d u$, var. cariosum, Sull.,

Menard.

Fulton, Menard. Menard.

Fulton, Menard.

6 .

$66 \quad 66$

$66 \quad 66$

$66 \quad 66$

$66 \quad 66$

Ill.

Fulton, Menard. Fulton, Menard. Menard, Fulton. Fulton, Menard. 6646 66

Fulton, Menard, S. Ill. Fulton. 66

Fulton, Menard.

$\begin{array}{lll}66 & 66 \\ 66 & 6 & \\ 66 & 66 \\ 66 & 66 & \\ & & \text { Ill }\end{array}$

\section{HEPATICAE.}

RICCIACEAE.

Riccia, Miche

R. sorocarpa, Biscl.,

R. lescuriana, Aust.,

R. natans, $L$.,

R. lutescens, Schwein.,

R. fluitans, $L$,

var. canaliculata, Hoffm.,

var. sullivanti, Aust.,

Fulton, Menard.

R. frostii, Aust.,
"6 6
6646
6. 66
Fulton. 66

Menard.

Fulton. 
ANTHOCEROTEAE.

Anthoceros, Mich.
A. punctatus, $L$.,
A. laevis, $L$., var. major,
A. orbicularis, Schuein.,
Fulton, Menard. Fulton, Menard, Union. Fulton. 66

MARCHANTIACEAE.

Marchantia, $L$.

I. polymorpha, $L$.,

Conocepialus, Hitl.

C. conicus, $L$,

Fulton, Menard, Union.

Asterella, Beauv.

A. hemisphaerica, L.,

D. barbifrons, Biseh.,

F. tenella, Nees, Fulton, Menard.

JUNGERMANNIACEAE.

Aneura, Dumort.
A. pinguis, $L$.,
A. sessilis, Spreny.,
A. multifida, L.,
A. latifrons,

Dutalia, Nees.

Fimbriaria, Nees.

Fulton, Menard.

Fulton, Menard, Union.

Fulton, Menard. 
Blepharostoma, Dumort.

B. trichophyllum, $L$.,

Fulton.

Cephalozia.

C. sullivantiae, Aust.,

C. divaricata, Engl. Bot.,

Jobnson.

Fulton.

66

C. bicuspidata, I.,

C. curvifolia, Dicks.,

C. connivens, Dicks.,

C. albescens, Hook.,

Fulton, Menard, Union, Johnson.

IIl.

Harpanthus, Nees.

H. scutatus, Mitt.,

Union, Johnson

Jungermannia, $L$.

J. schraderi, Mart.,

J. hyalina, Lyell,

Fulton, Menard, Union Johnson.

Fulton.

LEPTOSCYPHUS.

L. taylori, Hook.,

Fiulton.

Scaphania, Lindenberg.

S. nemorosa, $L$.,

Johnson.

Frullania, Raddi.

F. grayana, Mont.,

Menard.

S. 111.

F. squarrosa, Nees,

F. aeolotis, Nees,

F. virginica, Gottsche,

F. eboracensis, Gottsche,

Fulton, Menard.

66

66

66

66

Madotheca, Dumort.

M. thuja, Dicks.,

M. porella, Dicks.,

R. complanata, $L$.,

Fulton, Menard.

"6 6

$$
\text { Radula, Nees. }
$$

Blepharozia.

B. ciliaris, $L$.,

Fulton, Menard.

Calypogeia, Raddi.

C. trichomanis, Dicks.,

Menard. 


\section{LICHENES.}

USNEEI.

Ramalina, Ach.

R. calicaris, $F r$.,

Fulton, Menard, S. Ill. Fulton, Menard.

var. fraxinea, $F r$,

Cetraria, Ach., Fr.

U. cilaris, Ach.,

Fulton, Henard.

Usnea, Ach.

U. barbata, (L.) Fr., var. florida, $F r$, var. strigosa, Ach., var. rubiginusa, Mich.,

Fulton, Ienard, S. Ill. Menard. Fulton. Menard.

Alectoria, (Ach.) Nyl.

1. jubata, (L.) Fr, var. chalybeiformis, Ach.,

Fulton.

\section{PARMELIEI.}

Theloschistes, Norm., Tuck.

T. parietinus, (L.) Norm.,

T. concolor, (Diclis, )

Menard. Fulton, Menard.

Parmelia, (Ach.,) D. N.

P. perforata, Ach., var. crinita, (Ach.) Tuck.,

P. perlata, (L.) Acl., var. olivetorum, $A c h$.,

P. tiliacea, (Hoffm.) Flk.,

P. borreri, Turn., var. rudecta, Tuck,

P. saxatilis, (L.) Fr., var. rosa eformis, Ach.,

P. laevigata, Ach.,

P. aurulenta, Tuck,

P. colpodes, Ach.,

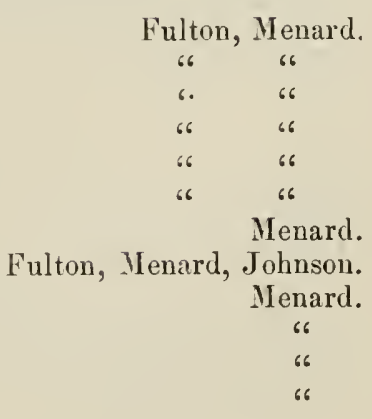


P. caperata, (L.) Ach.,

Fulton, Menard, S. Ill.

P. conspersa, (Ehrh.) Ach., var. stenophylla, Ach.,

P. olivacea, (L.) Ach. var, aspidota, $A c h$. Fulton, " " Menard.

Physcia, (Fr.) Th. $F r$.

P. aquila, (Ach.) Nyl. var. detonsa, Tuck.,

P. pulverulenta, (Schrel.) Nyl.,

P. speciosa, (Wulf. Er.) Nyl., var. hypoleuca, Ach.,

P. stellaris, $(L) N y$.$l .,$ var. tribacia, $F r$,

P. cæsia, (Hoffm.) Nyl., var. stellata, $F r$.,

P. obscura, (Ehrh.) Nyl., var. ciliata, Schaer., var. agglutinata, Schaer.,

Pyxine, $F r$.

P. cocoes, $(S w) ., N y l$. var. sorediata, Tuck.,

Union. Fulton, Menard, S. Ill. 66 Fulton, Menard. $66 \quad 66$

$66 \quad 66$

Fulton. Fulton, Menard.

66 66 66 66 66

PELTIGEREI.

Sticta, (Schreb.,) Del.

S. herbacea, Ach., (?)

Menard.

Nephroma, Ach.

N. laevigatum, Ach.,

Union, Johnson.

Peitigera, (Hoffm.) Fee.

P. canina, (L.) Hoffm.,

P. polydactyla, (Neck.) Hoffm.,

Fulton, Menard. 66

PANNARIEI.

Heppia, Naeg.

H. despreauxii, Mont.,

Fulton, Menard.

Pannaria, (Del.) Tuck.

P. microphylla, (Sw.) Del.,

P. leucosticta, Tuck.,

Union, Johnson 
P. crossophylla, Tuck,

P. molybàaea, Pers, var, cronia, Tuck.

S. Ill.

Fulton, Johnson.

P. nigra, (Huds.) Nyl.

(Sonthern specimens in finit,)

P. byssina, (Iloffm.) Tuck.,

Fulton, Menard, S. Ill.

Fulton, Menard.

COLLEMEI.

Ephebe, $\left(F^{\prime} r.\right)$ Tuck.

E. pubescens, $F r$,

Menard.

Synalissa, $F r$.

S. schaereri, Muss.,

S. phaeococca, Tuck.,

Fulton, Nenard, S. Ill.

Fulton.

Collema, (Hoffm.) Fr.

C. pycuocarpum, $N y l$,

C. cyrtaspis, Tuck.,

C. nicrophyllum, Ach.,

C. verruciforme, Ach.,

C. leptaleum, Tuck.,

C. flaccidum, Ach.,

C. nigrescens, (Huds.) Ach.,

C. pulposum, (Bernh.)

$$
\text { var. tenax, Ach., }
$$

C. limosum, Ach.,

C. arenosum, (Trulf.) Schaer.,

C. pustulatum, Ach.,

Fulton, Menard.

"6 6

Menard, Union Mlenard. 66

Fulton, Johnson.

Fulton, Menard.

Fulton, Menard, Johnson.

III.

Fulton, Menard. Menard.

Johnson.

Leptogiun, Fr.

L. subtile, $N y l$.,

L. minutissinum, Flk.,

L. lacerum, (Su.) Fr., var. bolacinum, Schaer.,

L. pulchellum, (Ach.) Nyl.,

L. tremelloides, $F r$.,

L. cresiellum, Tuck., ined. n. sp.,

L. chloromelum, (Sx.) Nyl.,

L. myochroum, (Ehrh.) Schaer., var. saturninum, (Dicks.) Tuck.,

L. dactylinum, T'uck.,
Fulton, Menard, Union. Menard. l'ulton, 66

Fulton, Menard, Johnson. Fulton, Menard.

66 $66 \quad 66$

S. Ill.

Johnson.

Fulton. 
P. sideritis, Tuck.,

P. vitellinum, (Ehr.h.) Hepp.,

P. cerinum, (lledw.) Naeg.,

P. aurantiacum, (Light.) Naeg.,

P. ferrugineum, (Huds.,) Hepp., var. nigricans, Tuck.,

P. camptidium, Tuck.
Fulton.

Fulton, Menard. Fulton, Menard, S Ill. Fulton, Menard, Johnson.

Fulton, Menard, S. Ill. Fulton. Fulton, Menard.

J.ecanora, Arh., Tueti.

L. muralis, (Scherel.) Schaer.,

S. Ill. 66

L. tartarea, (L.) Ach.,

I. subfusca, (L.) Ach., var. discolor, $F r$.

I. varia, (Ehrh.) Fr., var. aitema, Ach., var. sarcopis, Wahl.,

L. elatina, Ach., var. ochrophaea, Tuck.,

Fulton, Menard, Union, Johnson. Fulton. Fulton, Menard.

Fulton. ،

L. cinerea, $L$.

S. Ill.

L. cervina, Pers., var. eucarpa, $、$ yyl, var. pruinosa, Ach.,

Rinodina, Mass. var. confragosa. $N y l$.,

R. ascociscana, Tuck.,

R. albo-atra, $F$.

R. constans, (Nyl.) Tuck.,
R. sophodes, (Ach.) Mass.,

Fulton, Menard. Fulton, Johnson. Fulton, Menard.

IIl.

Fulton.

Pertusaria, D. $C$.

P. pertusa, (L.) Ach.,

P. leioplaca, (Ach.) Schaer.,

P. velata, (Turn.) Nyl.,

P. pustulata, (Ach.) Nyl.,

P. wulfenii, $D C$.,

P. globularis, Ach.,

Menard, Johnson. Fulton, Menard.

$\begin{array}{ll}66 & 66 \\ 66 & 66 \\ 66 & 66 \\ & \text { S. Ill. }\end{array}$

Conotrema, Tucl.

C. ureeolatum, (Ach.) Tuck.,

Fulton, Menard.

Gralecta, Ach.

G. pineti, (Schrad.) Fr.,

G. trivialis, Willey, ined.n.sp., 
CLADONIEI.

Cladonta, Hoffim.

C. alcicornis, $F r$,

Johnson.

C. pyxidata, $F r$, var. symphyearpa, Fr.,

C. eariosa, (Ach.) Spreny.,

C. fimbriata, ( $\left.I_{\text {. }}\right) \mathrm{Fr}$., var. tubaeformis, Hoffm., var. adspersa, Tuck.,

C. gracilis, (L.) Fr., var. verticillata, $\mathrm{Fr}$.,

C. mitrula, Tuck.,

C. turgida, (Ehrh.) Hoffm.,

C. furcata, (Huds.) $F r$. var. crispata, Fll., var. racemosa, Flli., var. subulata, Fll...,

C. squamosa, Hoffm., var. delicata, $F r$. rar. caspiticia, $N_{y} l$, ,

C. rangiferina, (L.) Hoffm.,

C. uncialis, (L.) Fr.,

C. maeilenta, Hoffm.,

C. museigena, Eschw.,

C. cristatella, Tuck.,

var. ramosa, Tuck.,

Fulton, Menard.

Menard.

Fulton.

Fulton, Menard.

Fulton.

Fulton, Menard.

$66 \quad 66$

$66 \quad 66$

$66 \quad 66$

$66 \quad 66$

Fulton, Menard, Johnson, Union.

Fulton, Menard.

Menard.

66

Fulton, Menard.

$66 \quad 66$

Johnson.

Fulton, Menard, Johnson.

Johnson

Fulton, Menard.

Menard

Fulton, Meuard.

66

LECIDEEI.

BiATORA, F'.

B. rufo-nigra, Tuck.,

B. coaretata, $T h . F r$.,

13. flexuosa, Fr.,

B. parvifolia, Pers.,

B. russula, (Ach.) Nront.

B. sanguineo-atra, (Fr.) Tuck.,

B. carnulenta, Tuck.,

B. exigua, (C'laub.) Fr.,

B. nliginosa, (Schrad.) Fr.,

B. rudis, Willey,

B. peliaspis, Tuck.,

B. atro-purpurea, (Mass.) Tick.,

B. hypnophila, (Turn.) Tuck.,

S. Ill.

Fulton.

66

S. Ill

Fulton.

Fulton, Menard.

Fulton.

Fulton, Menard, Union.

Fulton, Menard.

Fiulton.

66

66

Fulton, Menard. 
B. rubella, (Ehrh.)

var. spadicea, Ach.,

var. suffusa, $F r$.,

var. intindata, Fr.,

B. umbrina, (Ach.) Tuck., var. bacillifera, Nyl., f. muscorum, Sev., "

B. chlorosticta, Tuck.,

B. chlorantha, Tuck.,

B. campestris, $F r$.

B. fossarum, (Duf.) Mont.,

B. cyphalea, Tuck.,

B. geophana, $N y l$.

B. resinac, $\mathrm{Fr} .{ }^{*}$,

Fulton, Menard, Union. Fulton, Menard.

- $66 \quad 66$

Fulton.

Johnson.

Fulton.

Fulton, Menard.

"6

$66 \quad 66$

Fulton.

Fulton, Menard.

Lecidea, $(A c h$.

L. albo-coerulescens, $F r$.,

1. enteroleuca, $\mathrm{Fr}$.,

L. tessellina, Tuck.,

L. myriocarpoides, $N y l$.,

Buellia, (D. N.) Tuck.

B. lactea, Mass.,

B. atro-alba, $(F l$.) Th. $F r$., var, chlorospora, $N y l$,

B. parasema, (Ach.) Kbr.,

B. myriocarpa, $(D C$.$) Madd.,$

Union.

Menard, Johnson.

S. Ill.

Fulton.

S. III.

Fulton, Menard.

Fulton.

OPEGRAPHEI.

Opegrapha, (Humb.) Ach., Nyl.

O varia, (Pers.) $F r$.,

Fulton, Menard.

O. vulgata, (Ach.) Nyl.,

Union

Graphis, Ach., Nyl.

G. seripta, (L.) Ach.,

G. eulectra, Tuck,

G. dendritica, Ach.,

Fulton, Menard, Union.

Menard.

Fulton, "

\section{ARTHONIEI.}

Arthonia, Ach. Nyl.

A. pyrrhula, $N y l$.,

A. lecideella, $N y l$.,

A. patellulata, $N y l$,

A astroidea, (Ach.) Nyl. var. epipasta, $N y l$.,

Fulton, Menard.

'6 66

Fulton.

Fulton, Menard.

*"Probably a fungus." - Willey. 
A. punctiformis, Ach.,

Fulton, Menard, Union.

A. polymorpha, Ach.,

Fulton.

A trediosa, $N y l$.,

A. spectabilis, Fl.,

I. pyenocarpum, $N y l$,

Fulton, Menard, Union.

\section{CALICIEI.}

Acoliun, ( Fee.) D N.

A. tigillare, $(A c h) D$.$N .$

Menard.

Calicium, Pers. Ach. Fr.

C. subtile, Fr.,

C. trachelinum, Ach.,

C. curtum, Turn. \& Borr.,

C. roscidum, Flli., var. trabinellum, Nyl., var. dosodes, Tuck., inerl.,

C. mierocephalum, (Sm.) Tum. \& Borr.,

C. populneum (?), DeBrogn.,

C. tubiforme, Ilass.,

C. turbinatum, Pers.,

Fulton, Menard.

“6

$$
\text { "6 }
$$

Fultor.

66

66

66

S. III.

Johnson.

Fulton.

Coniocrbe, Ach.

C. pallida, (Pers.) Fr.,

\section{ENDOCARPEI.}

Endocarpon, IIchio. $F r$.

E. miniatum, $(L .$,$) Schaer.,$ var manitense, Tuck.,

E. arboreum, Schwein.,

E. cinereum, Nees.,

E. rufescens, Ach.,

E. hepaticum, Ach.,

E. pusillum, Hedwo,

Union.

Johnson.

Fulton, Menard.

Fulton.

Fulton, Menard.

S. III.

Fulton, "6

\section{VERRUCARIEI.}

Segestria, $F r$.

S. lauveri, (Fl.) Tuck. Found on stones, rails and bark. Fulton, S. Ill. 
Staurothele, Norm.

S. diffractella, $(N y l$.$) Tuck.,$

S. Ill.

Sagedia, (Mass. Kbr.) Tuck.

S. cestrensis, Tuck.,

S. lactea, $K b r$.,

S. Ill. Fulton.

Verrucaria, (Pers.) Tuck.

V. epigæa, (Pers.) Ach.,

V. nigrescens, Pers.,

V. fuscella, Fr.,

V. rupestris, Schrad.,

V. muralis, Ach.,

V. pyrenophora, (Ach.) Nyl.,

Pyrenula, Ach.

P. thelena, (Ach.) Tuck., var. micula, $F l$.,

P. punctiformis. (Ach.) Nueg.,

P. nitida, Ach.,

P. subcinerea, (Nyl.) Tuck.,

P. gemmata, (Ach.) Naeg.,

P. leucoplaca, (Walbr.) Kbr.,

P. glabrata, (Ach.) Mass.,

P. lactea, (Mass.) Tuck,

Fulton, Menard.

Fulton.

S. Ill.

Fulton, "6

Adams.

Fulton.

Fulton, Menard.

Fulton.

Fulton, Menard.

Menard, Fulton. 66

66

Fulton, Menard. Fulton, Union. Fulton, Menard. 


\section{INDEX TO GENERA.}

Acaulon................... 18

Acolium.................... 33

Alectoria................... 27

Anacamptodon............... 23

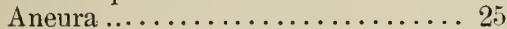

Anomodon.................. 22

Anthoceros ................. 25

Aphanorrhegma............... 22

Archidium.................. 18

Arthonia ................... 32

Asterella ................... 25

Astomum. ................ 19

Atrichium ................ 21

Aulacomnion ................ 21

Barbula..................... 20

Bartramia....................21

Biatora............................ 31

Blepharostoma............... 26

Blepharozia................. 26

Bruchia..................... 19

Bryum ................... 21

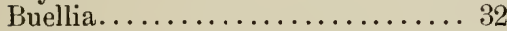

Calicium .................. 33

Calypogeia................. 26

Campylopus ................ 19

Cephalozia.................. 26

Ceratodon ................... 19

Cetraria................... 27

Chiloscyphus................ 25

Cladonia................... 3I

Clasmatodon................. 22

Climacium................... 23

Collema................... 29

Conocephalus ................. 25

Conomitrium................. 19

Conotrema ................. 30

Cylindrothecium.............. 23

Desmatodon ................. 20

Dichelyma................. 22

Dicranum ................. 19

Didymodon ................. 20

Discelium................... 22

Drummondia ................. 20

Duvalia.................... 25

Encalypta................... 20

Endocarpon.................. 33

Ephebe..................... 29

Ephemerum ................. 18

Fabronia ................. 23

Fimbriaria................. 25

Fissidens.................. 19

Fontinalis ................. 22

Frullania................... 26

Fmnaria..................... 22

Graphis................... 32

Grimmia................... 20

Gyalecta................... 30
Harpanthus. ............... 26

Hedwigia................... 21

Heppia.................... 28

Homalothecium.............. 23

Hypnum ................... 23

Jungermannia . . . . . . . . . . . . 26

Lecanora................... 30

Lecidea................... 32

Leptodon.................... 22

Leptogium ................. 29

Leptoscyphus................. 26

Leskea..................... 22

Leucobryum.................. 19

Leucodon................... 22

Lophocolea ................. 25

Madotheca ................. 26

Marchantia................. 25

Mnium ................... 21

Mycoporum ............... 33

Nephroma................. 28

Opegrapha .................. 32

Orthotrichum................ 20

Pannaria.................. 28

Parmelia.................... 27

Peltigera ................... 28

Pertusaria................. . 30

Phascum.................... 18

Physcia .................. 28

Physcomitrium............... 22

Placodium.................. 30

Platygyrium................ 23

Pleuridium ................ 18

Pogonatum................. 21

Polytrichum.................. 21

Pottia...................... 20

Ptychomitrium............... 20

Pylaisaea................... 23

Pyrenula.................... 34

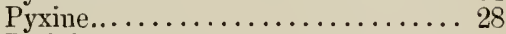

Radula................... 26

Ramalina ................. 27

Riccia.................... 24

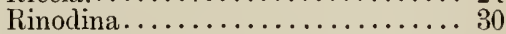

Sagedia................... 34

Scaphania.................. 26

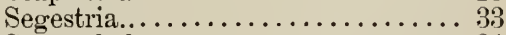

Staurothele................ 34

Sticta.................... 28

Synalissa ................... 29

Tetraphis ................... 20

Thelia.................... 2:3

Theloschistes .............. 27

Timmia.................... 21

Trematodon .................. 19

Trichostomum................. 20

Usnea..................... 27

Verrucaria.................. 34

Weisia ................... 19 
